\title{
Short-Term Memory of the Amplitude of Body Rotation in Orienting Behavior of African Clawed Frog (Xenopus laevis)
}

\author{
Gouki Okazawa' ${ }^{1}$ and Shintaro Funahashi ${ }^{1,2,3}$ \\ ${ }^{1}$ Faculty of Integrated Human Studies, Kyoto University, Sakyo-ku, Kyoto 606-8501, Japan \\ ${ }^{2}$ Department of Cognitive and Behavioral Sciences, Graduate School of Human and Environmental Studies, Kyoto University, \\ Sakyo-ku, Kyoto 606-8501, Japan \\ ${ }^{3}$ Kokoro Research Center, Kyoto University, Sakyo-ku, Kyoto 606-8501, Japan \\ Correspondence should be addressed to Shintaro Funahashi; funahashi.shintaro.2z@kyoto-u.ac.jp
}

Received 11 February 2013; Accepted 28 February 2013

Academic Editors: A. Arslan, I. Krams, D. Park, and A. Robins

Copyright (c) 2013 G. Okazawa and S. Funahashi. This is an open access article distributed under the Creative Commons Attribution License, which permits unrestricted use, distribution, and reproduction in any medium, provided the original work is properly cited.

African clawed frog (Xenopus laevis) can orient its body toward the prey by analyzing the direction of approaching water waves. Xenopus accurately orients toward the source of the stimulus when the stimulus is generated several $\mathrm{cm}$ away from its body. However, although Xenopus orientation behavior fluctuates when the stimulus is generated very near or above its body, the amplitude of the body rotation in the orienting behavior was affected by the preceding orienting behavior that had been performed several seconds before. In particular, the amplitude of the rotation in response to the stimulus applied above the body was positively correlated with that of the preceding rotation behavior in response to a stimulus generated several $\mathrm{cm}$ away from the body, indicating that Xenopus tends to repeat the preceding behavior if the direction of the stimulus is ambiguous. The results presented show the evidence that Xenopus can retain the amplitude of the rotation of the preceding orienting behavior for several seconds.

\section{Introduction}

African clawed frogs (Xenopus laevis) capture living insects on the water surface by analyzing the directions of water waves produced by the movement of the prey [1]. During this orienting behavior, water waves are predominantly detected by lateral lines that are dispersed over the frog's body. Lateral lines consist of 160-300 stitches, each of which contains hair cells (mechanoreceptors) $[2,3]$. When the frog detects water waves produced by the prey using lateral lines, it rapidly orients its body toward the source of the waves. Characteristics of this orienting behavior by African clawed frogs have been analyzed in many previous studies [4-9]. Elepfandt [4] first analyzed the accuracy of African clawed frog's orienting behavior and showed that African clawed frogs could accurately orient their bodies toward the direction of the wave source. In this study, the distance between the wave source and the frog's body was nearly constant (10$12 \mathrm{~cm}$ ). Subsequent studies have shown that African clawed frogs performed the orienting behavior accurately even if the distance between the frog's body and the wave source was altered $[8,9]$.

Although African clawed frogs performed the orienting behavior very accurately when the stimulus was presented several $\mathrm{cm}$ away from its body, it is unknown (1) how African clawed frog orients its body when the direction of the wave source is difficult to determine, such that the stimulus is generated very near or directly above its body, and (2) what parameters of the stimulus or frog's behavior affect frog's orienting behavior under this condition. In the present study, to address these issues, we examined frog's orienting behavior when the wave stimulus was applied directly above its body. We found that African clawed frogs tended to repeat the preceding orienting behavior under this condition. Moreover, we found that this tendency was maintained for several seconds after the termination of the preceding orienting behavior, indicating that African clawed frogs can retain some parameters of the preceding orienting behavior for several seconds. In this paper, we examined what parameters of the preceding orienting behavior the frog maintained and 
how long the frog maintained this information. A part of these results had been reported in abstract form [10].

\section{Methods}

2.1. Experimental Animals. The experiment was performed using 6 adult African clawed frogs (Xenopus laevis) with body lengths of $7.5-9.5 \mathrm{~cm}$ (snout to vent). Frogs were purchased from a local breeder (Shimizu Experimental Materials, Kyoto, Japan). They were kept in a water tank in $15 \mathrm{~cm}$ of water that was maintained at about $20^{\circ} \mathrm{C}$ and were fed with commercially available dry food once a day. Claas and Dean [9] reported that visual stimuli elicited orienting behaviors in frogs. Therefore, to exclude the effect of visual stimuli on the frog's orienting behavior, two frogs (F1 and M1) were blinded bilaterally by enucleation. For this procedure, frogs were anaesthetized by keeping them in a $0.1 \%$ solution of tricaine methane sulfonate (MS222) for more than $10 \mathrm{~min}$. After the frogs were anesthetized, both eyes were removed. We fully took care of these frogs for 1 week after the enucleation. Behavioral experiments for these two frogs were started at least one month after this procedure.

2.2. Experimental Setup. The experiment was performed in a PVC pool (the diameter was $60 \mathrm{~cm}$ and the water depth was $5 \mathrm{~cm}$ ). The frog was kept in this pool individually for at least 2 hours before the experiment to adapt to this environment. Water waves (stimuli) were generated by dipping a thin rod ( $3 \mathrm{~mm}$ in diameter) into the water. We continuously moved the rod up and down about $1 \mathrm{~cm}$ at $2-3 \mathrm{~Hz}$ by hand. The stimulus was applied when the frog was stationary in the pool. The frog's behavior was recorded by a PC camera (CMSV19, Sanwa, Tokyo, Japan), which was set $100 \mathrm{~cm}$ above the pool. A custom-made program using Java Media Framework (Oracle, Redwood Shore, CA, USA) controlled the camera. Average sampling rate was 12 frames/sec. From the recorded movie of the frog's orienting behavior, 2-3 images before the initiation of the frog's orienting behavior and 2-3 images after the termination of the frog's orienting behavior were taken for analysis. The termination of the orienting behavior was defined as the time when the frog stopped its rotation behavior and kept its body orientation steady for $1 \mathrm{sec}$. These images were analyzed with a custom-made program using Java to calculate the stimulus position in relation to the frog's body and the behavioral parameters of the frog's orienting behavior.

2.3. Stimulus and Behavioral Parameters. Figure 1 shows the methods to measure the stimulus position and behavioral parameters for this experiment. We used similar methods as those Claas and Dean [9] had used. The stimulus location (SL) is defined as the location where the wave stimulus was generated. The frog's body axis was determined as the line connecting the tip of the mouth to the center of the back. The rotation center (RC) is defined as the intersection of two axes: the frog's body axis before the initiation of the orienting behavior and the frog's body axis after the termination of the orienting behavior. In Figure 1, RC is

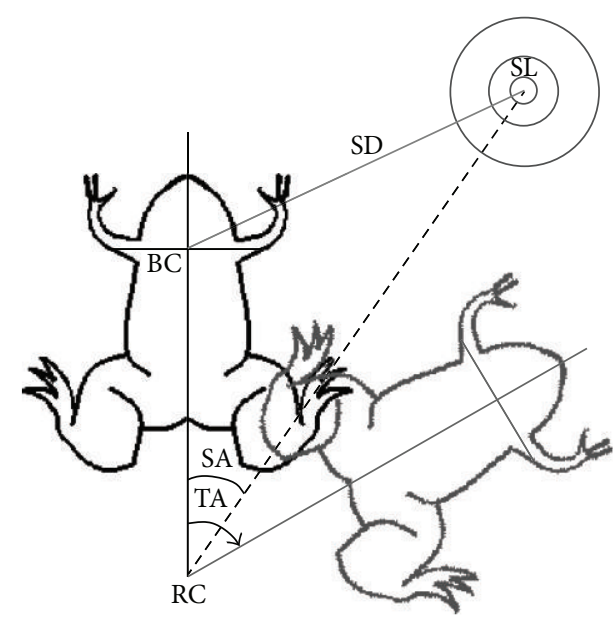

FIGURE 1: Parameters used in the present study. BC: body center; RC: rotation center; SA: stimulus angle; SD: stimulus distance; SL: stimulus location; TA: turn angle. Modified from Claas and Dean [9]. RC is placed outside of the frog's body to clarify the definition of RC. However, RC was located at the frog's pelvic region in most of orienting behaviors.

placed outside of the frog's body to clarify the definition of RC. However, RC was located at the frog's pelvic region in most of orienting behaviors. The stimulus angle (SA) and the turn angle (TA) were defined using the rotation center (RC). The stimulus angle (SA) was defined as the angle at $\mathrm{RC}$ between the frog's body axis before the initiation of the orienting behavior and the line connecting SL and RC. When the stimulus is generated on the line of the frog's body axis and when it is generated straight ahead of the frog's head, the stimulus angle was defined as $0^{\circ}$. Positive and negative stimulus angles were used to describe clockwise (rightward) and counterclockwise (leftward) directions of the stimulus against the frog's body axis, respectively. The turn angle (TA) was defined as the angle at RC between the frog's body axis before the initiation of the orienting behavior and the frog's body axis after the termination of the orienting behavior. Positive and negative values were used to describe clockwise (rightward) and counterclockwise (leftward) directions of the turning behavior, respectively. The turn amplitude was defined as the absolute value of TA. The body center (BC) was defined as the intersection between the frog's body axis and the line connecting the left and right shoulders. The stimulus distance (SD) was defined as the distance between BC and SL.

2.4. Experimental Procedure. To examine the frog's orienting behavior to the stimulus, two types of trials were introduced. In a "normal trial," the stimulus was applied more than $1.5 \mathrm{~cm}$ away from the frog's lateral surface. The mean distance between the stimulus and the frog's lateral surface was $9.0 \mathrm{~cm}$ for all frogs used. Stimulus locations (SLs) were selected randomly. In an "above-body trial," the stimulus was applied directly above the frog's pelvic region. Behaviors under normal trials were examined in all 6 frogs. Behaviors under above-body trials were examined in the 2 blind frogs. In this experiment, we tried to focus on the importance of the lateral 
TABLE 1: Number of trials in which the frogs turned to the right (clockwise) or the left (counterclockwise) in above-body trials $\left({ }^{*} P<0.05\right.$; ${ }^{* *} P<0.01 ;{ }^{* * *} P<0.001$, chi-square test).

\begin{tabular}{|c|c|c|c|c|c|c|}
\hline \multicolumn{7}{|c|}{ Stimulus location relative to the body axis } \\
\hline & \multicolumn{3}{|c|}{ Right } & \multicolumn{3}{|c|}{ Left } \\
\hline Turn direction & Right & Left & Correct & Right & Left & Correct \\
\hline Frog M1 & 70 & 37 & $65.4 \%^{* * *}$ & 49 & 52 & $51.5 \%$ \\
\hline Frog F1 & 37 & 23 & $61.7 \%^{*}$ & 16 & 43 & $72.9 \%{ }^{* * *}$ \\
\hline
\end{tabular}

The percentage under "correct" is the proportion of trials in which frogs made an orienting behavior toward the same direction as the stimulus direction.

TABLE 2: Similarity of turn amplitudes between a normal trial and a subsequent above-body trial with different intervals between these two trials.

\begin{tabular}{lcccc}
\hline & & $0-10 \mathrm{~s}$ & $10-20 \mathrm{~s}$ & $20-30 \mathrm{~s}$ \\
\hline \multirow{2}{*}{ Frog M1 } & $n$ & 92 & 73 & 45 \\
& Correlation & 0.512 & 0.353 & $(P=092$ \\
Frog F1 & $n$ & $(P<0.001)$ & $(P=0.002)$ & $(P=0.546)$ \\
& Correlation & 0.497 & 0.444 & \\
& & $(P<0.001)$ & $(P=0.003)$ \\
\hline
\end{tabular}

"Correlation" refers to the correlation between the turn amplitude in the normal trial and the turn amplitude in the subsequent above-body trial.

lines on the frog's orienting behavior toward potential baits. Therefore, we did not examine behaviors under above-body trials in the 4 normal frogs.

Water waves (stimulus) were applied when the frog was stationary in the pool. Water waves were applied continuously until the frog showed an orienting behavior. During the experimental sessions, a food reward was not given after the frog performed the orienting behavior. However, for the frog to produce the orienting behavior without reward during the experimental sessions, the frog was fed dry food as a reward during training sessions. Each experimental session lasted until the frog did not show any orienting behavior in response to the stimulus. Usually the experiment lasted for 20-30 min.

To examine (1) whether the frog can correctly orient its body toward the source of the stimulus when the stimulus is generated directly above its body and (2) what factors affect the frog's orienting behavior under this condition, an abovebody trial that was preceded by a normal trial was extracted for further analysis. An above-body trial (second trial) and the preceding normal trial (first trial) were considered as a set (a pair of trials). We compared parameters of orienting behavior (directions, turn angles, and turn amplitudes) between these two trials within a pair and analyzed their relation to the time interval between the two trials. The time interval between two trials was defined as the interval between when the orienting behavior was initiated in the first trial and when the orienting behavior was initiated in the second trial.

\section{Results}

3.1. Orienting Behavior in Response to the Stimulus Applied Away from the Frog's Body. A total of 1144 normal trials were obtained using 6 frogs. Figure 2(a) shows the spatial distribution of stimulus locations for normal trials when Frog M1 was a subject. Figures 2(b)-2(d) are scatter plots that show relations between the stimulus angles and the turn angles observed in normal trials in all 6 frogs (b), in Frog M1 (c), and in Frog F1 (d). If the frog oriented accurately toward the stimulus location, the turn angle would be equal to the stimulus angle. As shown in Figures 2(b)2(d), stimulus angles and turn angles were highly correlated ( $r=0.97-0.98)$, indicating that the frogs' orienting behavior toward the stimuli is very accurate. However, frogs tended to make smaller rotations as the stimulus angle increased. This tendency is consistent with the results obtained in previous studies [4-9].

3.2. Orienting Behavior in Response to the Stimulus Applied Just Above the Frog's Body. In above-body trials, frogs showed a completely different behavior from that observed in normal trials. Figures 3(a) and 3(b) show the distribution of stimulus locations in 263 above-body trials for Frog M1 (a) and 131 above-body trials for Frog F1 (b). The stimulus was mostly applied directly above the frog's pelvic region. Figures $3(\mathrm{c})$ and 3(d) show relations between stimulus angles and turn angles observed in these trials for Frogs Ml and Fl, respectively. In the most of orienting behaviors, RCs were located at the frog's pelvic region, which was almost identical position where the stimulus was applied. Therefore, a small change of the stimulus location sometime causes a large change of the stimulus angle.

The turn angles varied from trial to trial, although stimulus locations were approximately the same. The turn angles were distributed between $-120^{\circ}$ and $120^{\circ}$. Wide distribution of turn angles observed in above-body trials could be explained by small variation of stimulus locations. For example, stimuli applied slightly rightward (leftward) to the frog's body might cause the frog to rotate rightward (leftward). Therefore, we analyzed relationships between frogs' turn direction and stimulus locations relative to the body axis (Table 1). We calculated the number of trials in which frogs turned either to the right or the left in response to a stimulus that was 


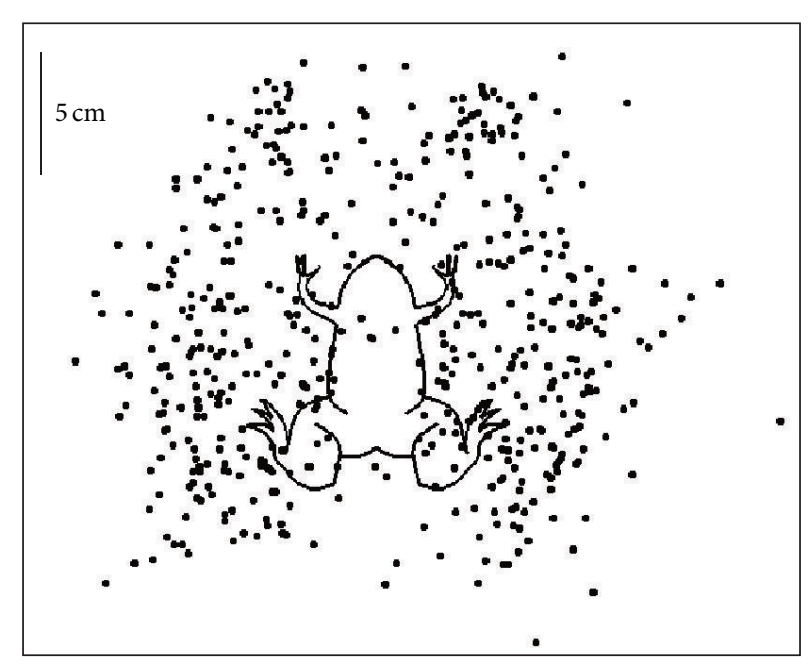

(a) Frog M1

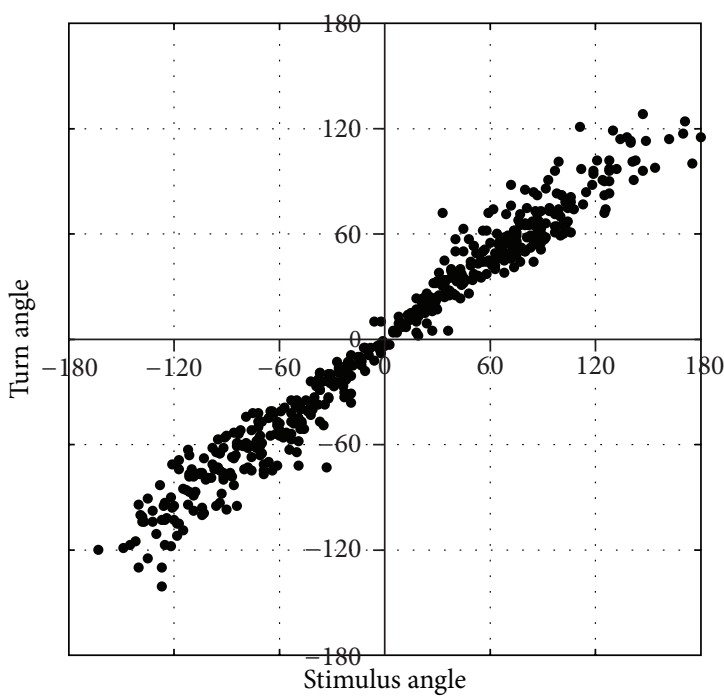

(c) Frog M1

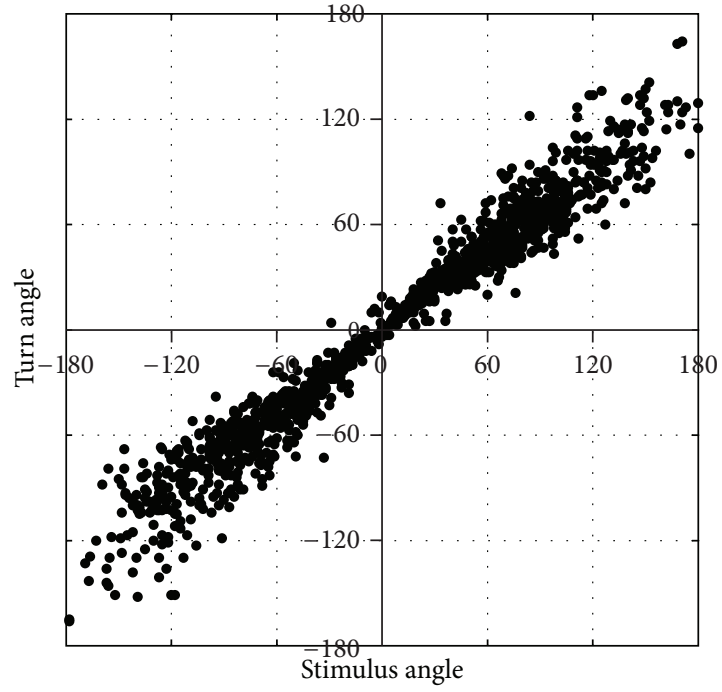

(b) All animals

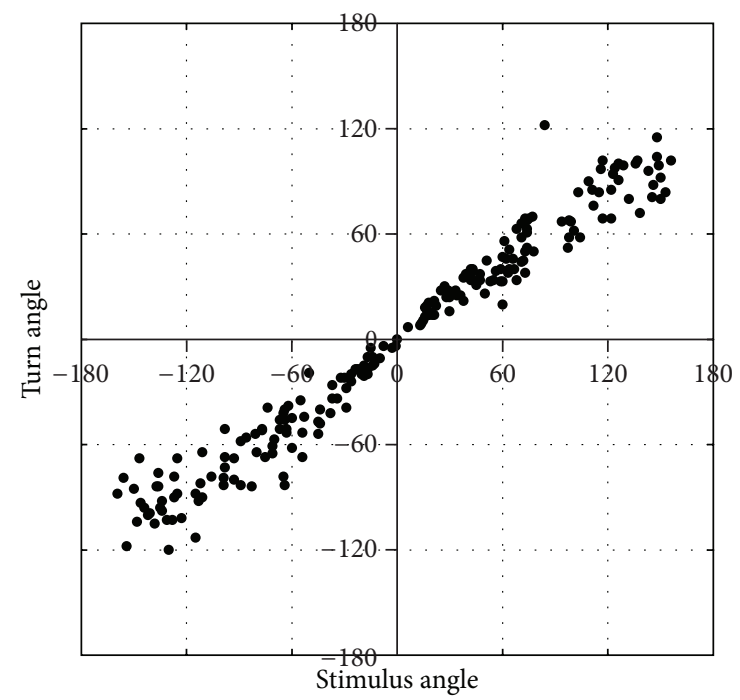

(d) Frog F1

FIGURE 2: (a) Spatial distribution of stimulus locations in normal trials for Frog M1. (b) Relation between stimulus angles and turn angles in normal trials for all 6 frogs. (c) Relation between stimulus angles and turn angles in normal trials for Frog M1. (d) Relation between stimulus angles and turn angles in normal trials for Frog F1.

applied at either the right or the left of the body axis in abovebody trials. Frog M1 turned to the right more often when the stimulus was applied on the right side $(P<0.001$, Chi-square test). However, Frog M1 turned equally to the right or the left when the stimulus was applied on the left side $(P=0.42$, chisquare test). On the other hand, Frog F1 tended to turn toward the direction where the stimulus was applied. Thus, while frogs' turn directions seemed to be affected by the stimulus locations relative to the body axis, their turn angles were not affected by the stimulus locations.

\subsection{Effect of the Preceding Orienting Behavior on the Frog's} Behavior in Above-Body Trials. In this experiment, both normal trials and above-body trials were introduced randomly for 2 frogs (M1 and F1) whose eyes had been surgically removed. During this procedure, we noticed that the turn direction of the frog's orienting behavior in the above-body trial was sometime similar to that of the orienting behavior in the preceding normal trial. To examine whether or not the turn direction in the above-body trial was affected by the direction of the orienting behavior in the preceding normal trial, we only examined above-body trials that were preceded by a normal trial within a $10 \mathrm{sec}$ time interval. Ninetytwo (Frog M1) and 76 (Frog F1) pairs of normal trials and subsequent above-body trials were extracted for this analysis.

Relations between the turn angles in normal trials and turn angles in subsequent above-body trials are shown in Figures 4(a) and 4(b). We found no significant relation in turn angles between preceding normal trials and subsequent above-body trials. However, as shown in Figures 4(c) and 


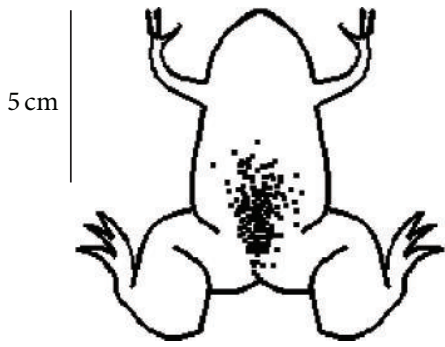

(a) Frog M1

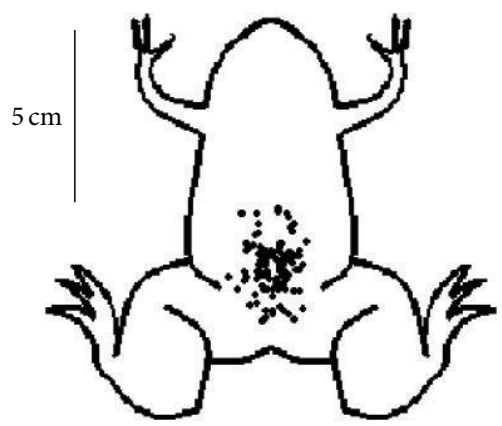

(b) Frog F1

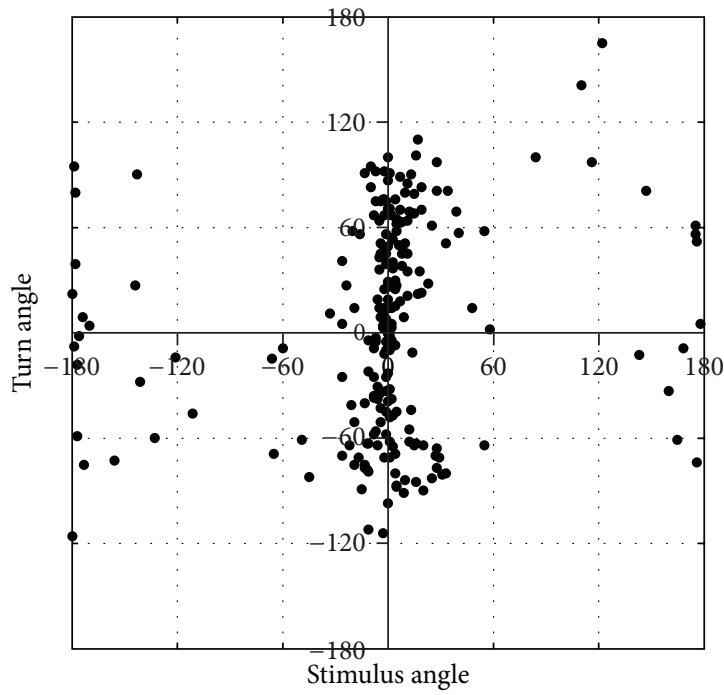

(c) Frog M1

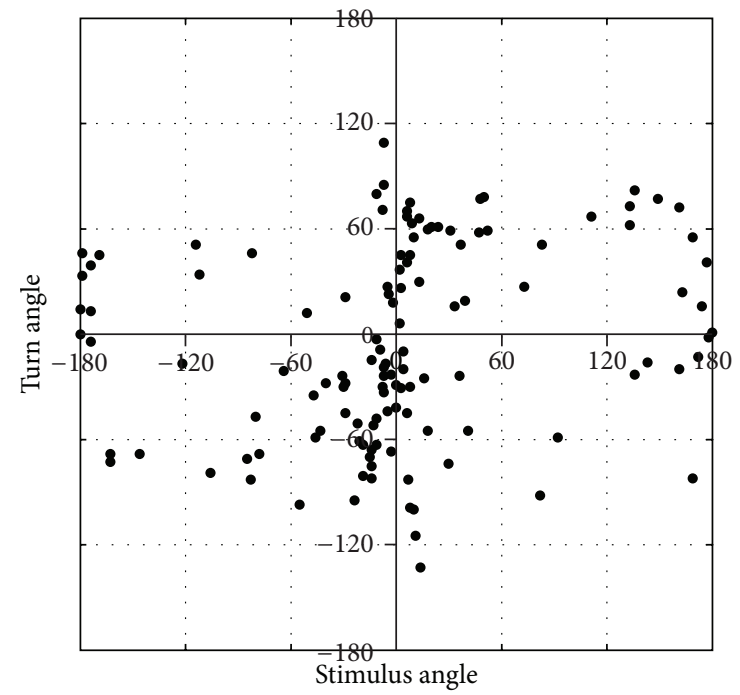

(d) Frog F1

FIGURE 3: Spatial distribution of stimulus locations in independent above-body trials for Frog M1 (a) and Frog F1 (b). Relations between stimulus angles and turn angles in independent above-body trials for Frog M1 (c) and Frog F1 (d). The stimulus angle and the turn angle were defined using the rotation center. In the most of orienting behaviors, rotation centers were located at the frog's pelvic region, which was almost identical position where the stimulus was applied. Therefore, a small change of the stimulus location sometime causes a large change of the stimulus angle.

4(d), significant positive correlations were found between turn amplitudes in normal trials and those in subsequent above-body trials in both frogs (Frog M1: $r=0.51, P<$ 0.001 ; Frog F1: $r=0.49, P<0.001)$. The slopes of the regression lines were significantly lower than 1.0 (Frog M1: slope $=0.73, P<0.001 ;$ Frog F1: slope $=0.79$, $P<0.001)$. These results indicate that both frogs tended to make rotations with smaller amplitudes in subsequent above-body trials compared with those in preceding normal trials, although frogs tended to make rotations with larger amplitudes in the subsequent above-body trials when frogs performed rotations with smaller amplitudes (less than $30^{\circ}$ ) in the preceding normal trials.

To evaluate the similarity of turn amplitudes between normal trials and subsequent above-body trials, the number of pairs was counted as a function of the difference in turn amplitude between the normal trial and the subsequent above-body trial (Figure 5). Black bars indicate trial pairs in which turn directions were the same between the above-body trial and the normal trial (same direction group). White bars indicate trial pairs in which the turn directions were opposite between the above-body trial and the normal trial (opposite direction group). For Frog M1, trial pairs whose difference of turn amplitude was less than $30^{\circ}$ consist of the major fraction (62 of 92 trial pairs, 67\%). In addition, trial pairs with the same turn direction also consist of the major fraction (40 of 62 trial pairs, 65\%). Similarly, for Frog F1, trial pairs whose difference of turn amplitude was less than $15^{\circ}$ were the major fraction (31 of 76 trial pairs, $41 \%$ ). However, for trial pairs whose difference of turn amplitude was less than $30^{\circ}$, trial pairs with opposite turn directions consist of the major fraction (30 of 51 trial pairs, $57 \%$ ).

Thus, when the wave stimulus is applied above the frog's body just after the termination of the orienting behavior, the frog produces an orienting behavior and this behavior is affected by the preceding orienting behavior. Frogs tend to make an orienting behavior with similar turn amplitude as that of the preceding behavior. This suggests that frogs maintain information regarding the turn amplitude of previously performed orienting behavior for some time. If the 


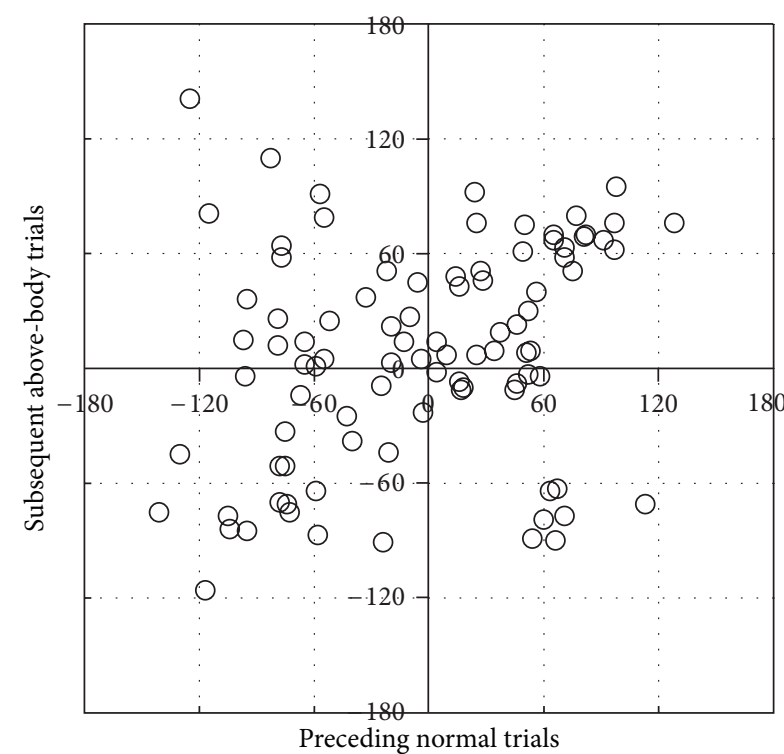

(a) Frog M1 (turn angles)

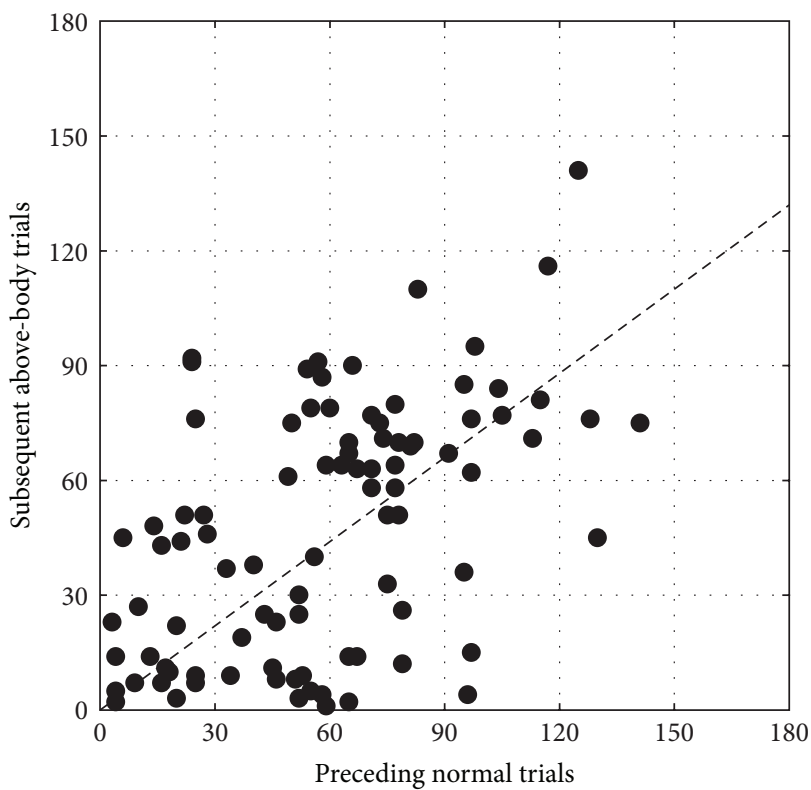

(c) Frog M1 (turn amplitudes)

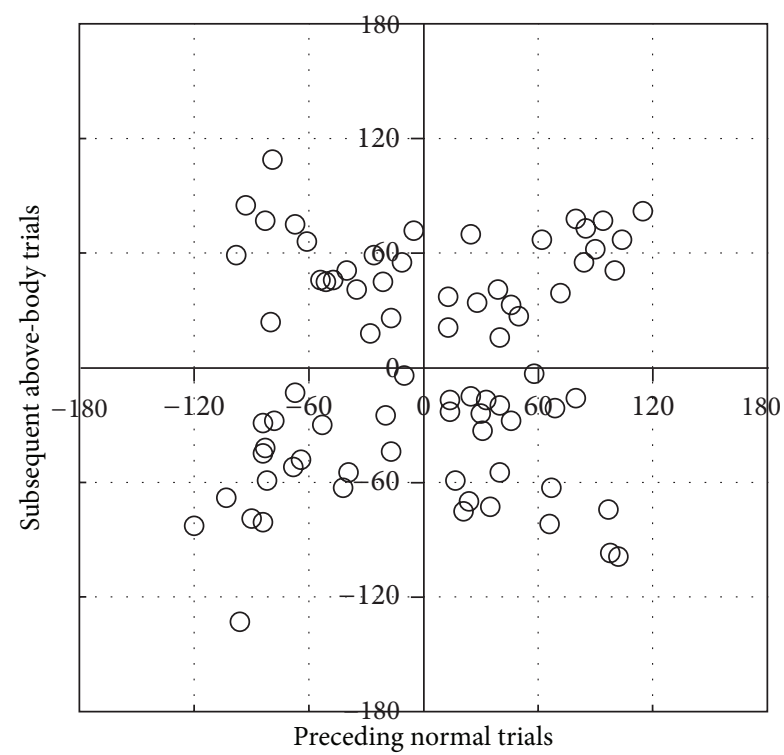

(b) Frog F1 (turn angles)

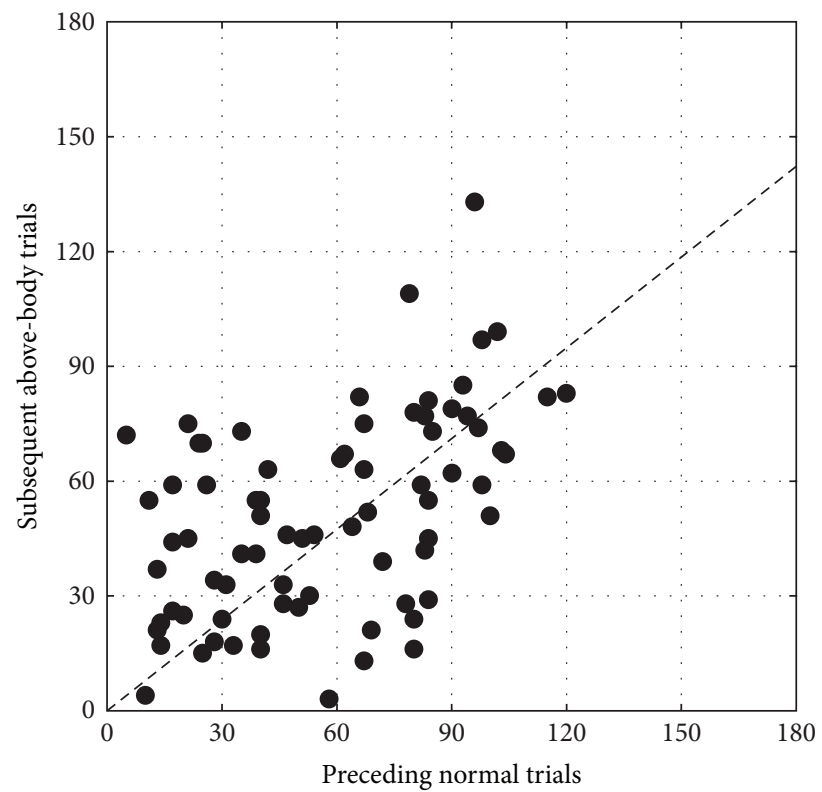

(d) Frog F1 (turn amplitudes)

Figure 4: (a) and (b) Relation of turn angles between preceding normal trials and subsequent above-body trials for Frog M1 (a) and Frog F1 (b). (c) and (d) Relation of turn amplitudes between preceding normal trials and subsequent above-body trials for Frog M1 (c) and Frog F1 (d).

next stimulus is generated within this time period and if the direction of the stimulus is difficult to determine, the frogs seem to make an orienting behavior with similar turn amplitude as that of the preceding behavior.

3.4. How Long Do the Frogs Retain the Information regarding the Preceding Orienting Behavior? To examine how long the frogs retained the turn amplitude of the orienting behavior performed in the preceding normal trial, we calculated the correlations between the turn amplitude in a normal trial and that in a subsequent above-body trial at intervals of up to $30 \mathrm{sec}$. Table 2 summarizes the correlations between turn amplitudes in normal trials and turn amplitudes in subsequent above-body trials. Turn amplitudes in normal trials and turn amplitudes in subsequent above-body trials were significantly correlated for both frogs, if the above-body trial was generated within $20 \mathrm{sec}$ after the initiation of the preceding normal trial. If the interval between the normal trial and the subsequent above-body trial became greater than $20 \mathrm{sec}$, significant correlation of turn amplitudes was not observed. These results indicate that the frogs could maintain information regarding the turn amplitude of the preceding orienting behavior for a short period of time $(<20 \mathrm{sec})$ and 


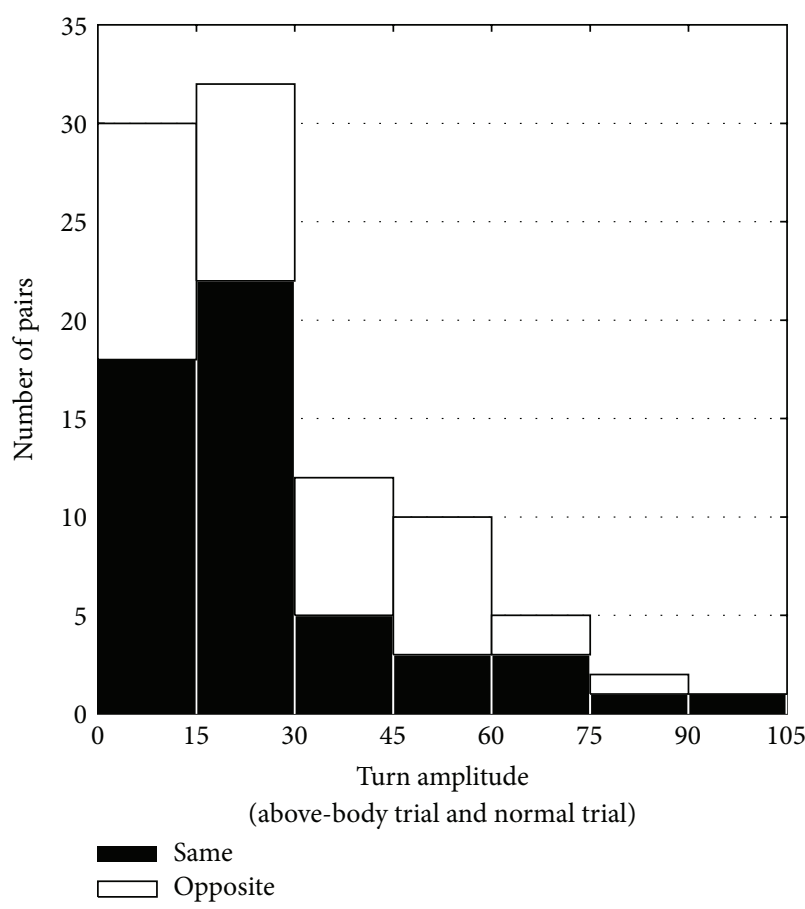

(a) Frog M1

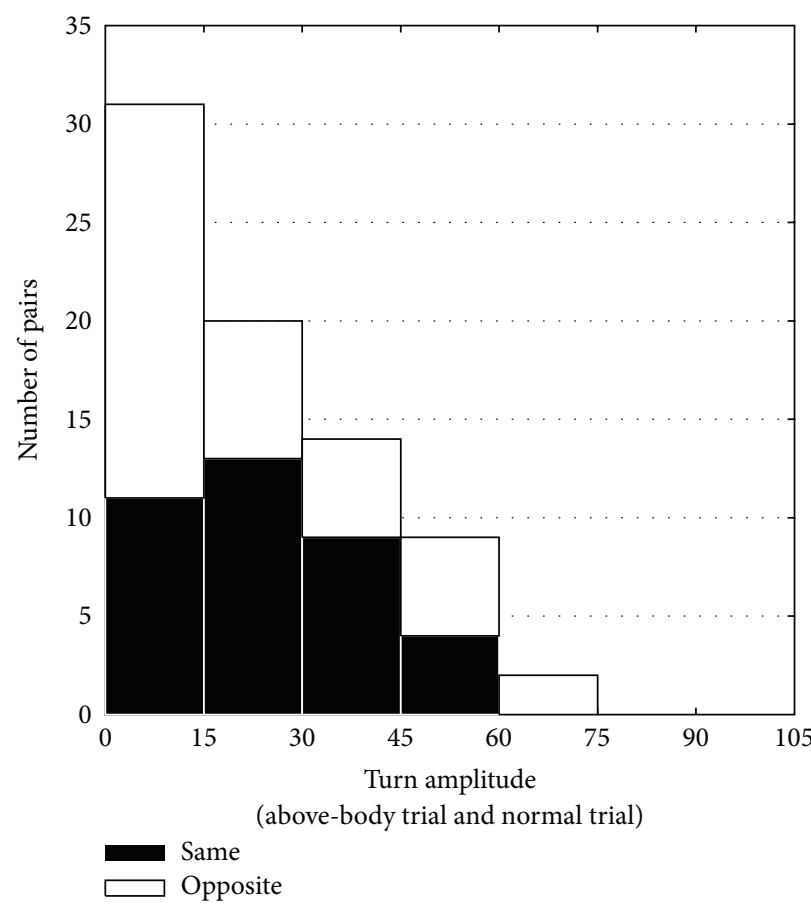

(b) Frog F1

FIGURE 5: Distributions of the differences in turn amplitudes between preceding normal trials and subsequent above-body trials for Frog M1 (a) and Frog F1 (b). Black bars indicate pairs of trials in which the direction of the orienting response in the above-body trial was the same as the direction in the preceding normal trial. White bars indicate pairs of trials in which the direction of the orienting response in the above-body trial was opposite to the direction in the preceding normal trial.

that this maintained information could be utilized to perform subsequent orienting behavior when the direction of the coming stimulus is difficult to identify for the frogs.

\section{Discussion}

4.1. Variation in the Response to a Stimulus Applied Above the Frog's Body. In this experiment, we first compared frog's orienting responses to wave stimulus generated directly above its body (above-body trials) with orienting responses to wave stimulus generated several $\mathrm{cm}$ away from its body (normal trials). For normal trials, the frogs' turn angles were proportional to the stimulus angles. This result is consistent with many previous studies [5-9]. On the other hand, the frogs' turn angles varied when the stimulus was applied directly above the frogs bodies. The turn angles were distributed widely between $-120^{\circ}$ and $120^{\circ}$. In above-body trials, the waves might activate lateral lines over the whole body with the similar time course and the similar strength. Therefore, synchronized activation of lateral lines at multiple body areas may make it difficult for the frogs to detect the direction of the incoming wave. Claas and Dean [9] had tested frog's orienting behaviors in response to waves generated at different distances from the frog's body. However, they did not observe large variation of turn amplitude when the stimulus was applied close to the frog's body. The difference between our results and theirs may be due to the difference in the body size of the frogs used for the experiment. Claas and Dean [9] used smaller frogs (body lengths of 2.8-4.8 cm) compared to the frogs that we used in this study (body lengths of 7.5-9.5 cm). A small distance between the frog's body surface and the wave source may cause a greater effect on orienting behaviors in smaller frogs compared with the effect observed in larger frogs. This may be the reason why different effects on frog's orienting behaviors were observed when the stimulus was applied close to the frog's body.

\subsection{Effect of the Preceding Orienting Behavior on Both Turn} Angles and Directions in Subsequent Above-Body Trials and Their Possible Mechanisms. In the present study, we found that the turn amplitude in the above-body trial was similar to that in the preceding normal trial, if the normal trial was followed by the above-body trial within the $20 \mathrm{sec}$ interval. Although the mean turn amplitude generated in above-body trials was smaller than that generated in preceding normal trials, the difference of turn amplitudes between above-body trials and preceding normal trials was less than $30^{\circ}$ in most cases we examined. This effect was maintained at least for up to $20 \mathrm{sec}$ after the initiation of the preceding normal trial. Since turn angles were predominantly determined by stimulus angles in normal trials, it could be concluded that this mnemonic effect was observed in above-body trials because, as the stimulus produced broad omnidirectional effect in sensory organs in above-body trials, it could be easy to estimate what information of the preceding trial affected behavioral parameters of the subsequent above-body trial 


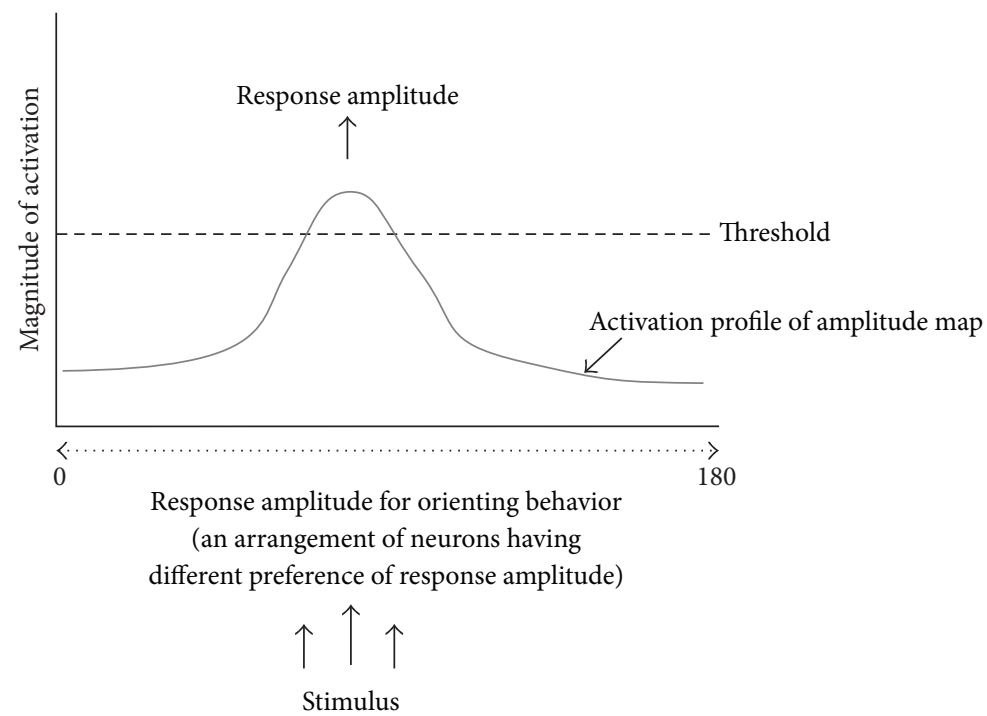

(a)

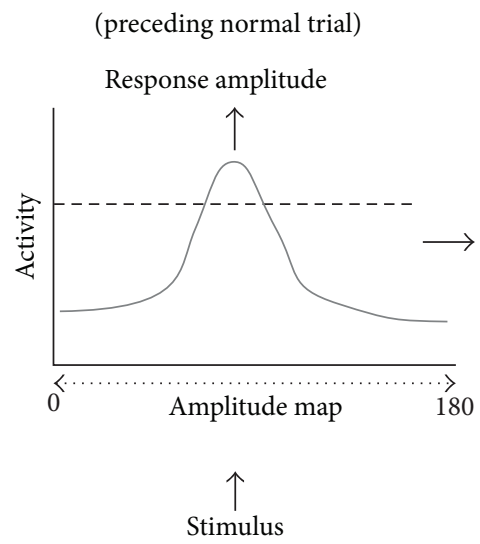

(intertrial interval)

(subsequent above-body trial)
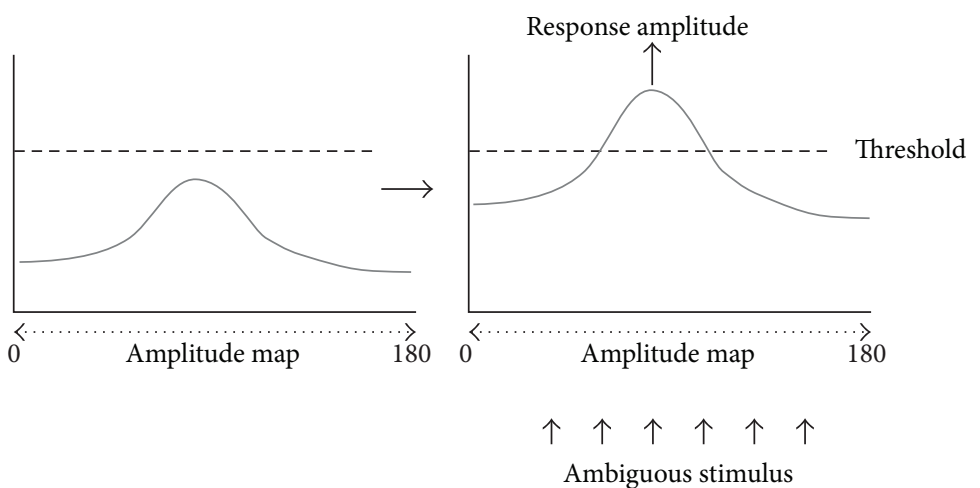

(b)

FigurE 6: Schematic drawings of a possible mechanism to explain the apparent retention of the preceding response. (a) Basic idea of the mechanism. (b) A possible mechanism that produces orienting responses in the above-body trial that have parameters similar to those in the preceding normal trial.

and how long this information was maintained after the termination of the preceding trial.

One possible mechanism to explain this phenomenon is shown in Figure 6(a). To produce a mnemonic effect in the orienting behavior, the brain area responsible for this phenomenon may have at least either sensory maps based on lateral-line organs distributed on the frog's body surface or motor maps to determine the amplitude of the rotation in the orienting behavior, although sensory systems other than lateral-line organs might be involved in the map formation [6]. When the water wave comes from a particular direction, only neurons with sensory or motor maps corresponding to the absolute value of the stimulus angle are activated (Figures 6(a) and 6(b), left). If the magnitude of activation of these neurons exceeds a threshold, the rotating behavior with that amplitude represented by those neurons could be generated. If the activation caused by the sensory stimulus is maintained in this group of neurons at a subthreshold level for some time after the termination of that behavior (Figure 6(b), middle) and if the next wave stimulates not just a small group of lateral-line organs but a large number of lateral-line organs on the frog's body (e.g., above-body trial), the large increase from the baseline activity might occur across most of the neurons in that brain area. If subthreshold activation caused by the previous stimulus is maintained in a group of neurons, the activation caused by the incoming stimulus might produce an additive effect on the maintained subthreshold activation and causes suprathreshold activation in this group of neurons (Figure 6(b), right). Thus, similar amplitude of the rotating behavior could be generated in the subsequent above-body trial as the preceding normal trial. Since this explanation is based on a pure speculation, 
neurophysiological study is needed to elucidate a mechanism for temporarily maintaining behavioral information of the preceding orienting behavior.

\subsection{Brain Areas That May Be Responsible for the Mnemonic} Effect. The present results indicate that some system temporarily maintains information regarding the previous orienting behavior. One possible mechanism is the sensitization of lateral-line organs. However, behavioral studies using frogs with lateral-line lesion $[4,5,8]$ and computational studies $[11,12]$ have indicated that a particular temporal pattern of lateral line activation is necessary to detect the wave direction. In addition, Zittlau et al. [13] showed that a single lateral line could not detect the wave direction. Therefore, the sensitization of lateral line organs may not be responsible for the mnemonic effect.

Information of the lateral line is sent to the lateral line nucleus, then to the cerebellum, and the torus semicircularis in the midbrain [14]. Elepfandt $[1,15]$ showed that a partial lesion of the torus semicircularis caused inaccurate orienting responses, suggesting that this area had a topographical map that represented the direction of the water wave. In addition, the optic tectum receives the output from the torus semicircularis and has similar topographic map as the torus semicircularis [13]. Therefore, the torus semicircularis and the optic tectum might be brain areas for the temporary maintenance of directional information. The optic tectum has reciprocal connections with the nucleus isthmi. The nucleus isthmi receive topographical inputs from the ipsilateral optic tectum and sends to the optic tectum [16-18]. This reciprocal network is thought to act as an attention-like mechanism to increase the frog's responsiveness to a particular direction [18]. Therefore, the reciprocal network between the optic tectum and the nucleus isthmi may also play a role in the mnemonic effect.

\section{Acknowledgment}

This study was supported in part by a Grant-in-Aid for Scientific Research (no. 17300103) from the Japanese Ministry of Education, Culture, Sports, Science, and Technology (MEXT) to S. Funahashi. Mr. G. Okazawa's current address is Division of Sensory and Cognitive Information, National Institute for Physiological Sciences, Okazaki, Aichi 444-8585, Japan.

\section{References}

[1] A. Elepfandt, "Central organization of wave localization in the clawed frog, Xenopus laevis. I. Involvement and bilateral organization of the midbrain," Brain, Behavior and Evolution, vol. 31, no. 6, pp. 349-357, 1988.

[2] A. Elepfandt, "Processing of wave patterns in the lateral line system parallels to auditory processing," Acta Biologica Hungarica, vol. 39, no. 2-3, pp. 251-265, 1988.

[3] C. Mohr and P. Görner, "Innervation patterns of the lateral line stitches of the clawed frog, Xenopus laevis, and their reorganization during metamorphosis," Brain, Behavior and Evolution, vol. 48, no. 2, pp. 55-69, 1996.
[4] A. Elepfandt, "Accuracy of taxis response to water waves in the clawed toad (Xenopus laevis Daudin) with intact or with lesioned lateral line system," Journal of Comparative Physiology A, vol. 148, no. 4, pp. 535-545, 1982.

[5] P. Görner, P. Moller, and W. Weber, "Lateral-line input and stimulus localization in the African clawed toad Xenopus sp.", Journal of Experimental Biology, vol. 108, pp. 315-328, 1984.

[6] B. Claas, H. Münz, and P. Görner, "Reaction to surface waves by Xenopus laevis Daudin. Are sensory systems other than the lateral line involved?" Journal of Comparative Physiology A, vol. 172, no. 6, pp. 759-765, 1993.

[7] B. Claas, "Removal of eyes in early larval stages alters the response of the clawed toad, Xenopus laevis, to surface waves," Physiology and Behavior, vol. 56, no. 3, pp. 423-428, 1994.

[8] B. Claas and H. Münz, "Analysis of surface wave direction by the lateral line system of Xenopus: source localization before and after inactivation of different parts of the lateral line," Journal of Comparative Physiology A, vol. 178, no. 2, pp. 253-268, 1996.

[9] B. Claas and J. Dean, "Prey-capture in the African clawed toad (Xenopus laevis): comparison of turning to visual and lateral line stimuli," Journal of Comparative Physiology A, vol. 192, no. 10, pp. 1021-1036, 2006.

[10] G. Okazawa and S. Funahashi, "Short-term memory observed in the orienting behavior of Xenopus laevis," Neuroscience Research, vol. 61, p. S117, 2008.

[11] J. M. P. Franosch, M. Lingenheil, and J. L. Van Hemmen, "How a frog can learn what is where in the dark," Physical Review Letters, vol. 95, no. 7, Article ID 078106, pp. 1-4, 2005.

[12] J. M. P. Franosch, M. C. Sobotka, A. Elepfandt, and J. L. Van Hemmen, "Minimal model of prey localization through the lateral-line system," Physical Review Letters, vol. 91, no. 15, Article ID 158101, 4 pages, 2003.

[13] K. E. Zittlau, B. Claas, and H. Münz, "Directional sensitivity of lateral line units in the clawed toad Xenopus laevis Daudin," Journal of Comparative Physiology A, vol. 158, no. 4, pp. 469-477, 1986.

[14] W. Plassmann, "Central neuronal pathways in the lateral line system of Xenopus laevis," Journal of Comparative Physiology A, vol. 136, no. 3, pp. 203-213, 1980.

[15] A. Elepfandt, "Central organization of wave localization in the clawed frog, Xenopus laevis. II. Midbrain topology for wave directions," Brain, Behavior and Evolution, vol. 31, no. 6, pp. 358368, 1988.

[16] M. J. Titmus, H. J. Tsai, R. Lima, and S. B. Udin, "Effects of choline and other nicotinic agonists on the tectum of juvenile and adult Xenopus frogs: a patch-clamp study," Neuroscience, vol. 91, no. 2, pp. 753-769, 1999.

[17] E. A. Dudkin and E. R. Gruberg, "Nucleus isthmi enhances calcium influx into optic nerve fiber terminals in Rana pipiens," Brain Research, vol. 969, no. 1-2, pp. 44-52, 2003.

[18] E. Gruberg, E. Dudkin, Y. Wang et al., "Influencing and interpreting visual input: the role of a visual feedback system," Journal of Neuroscience, vol. 26, no. 41, pp. 10368-10371, 2006. 

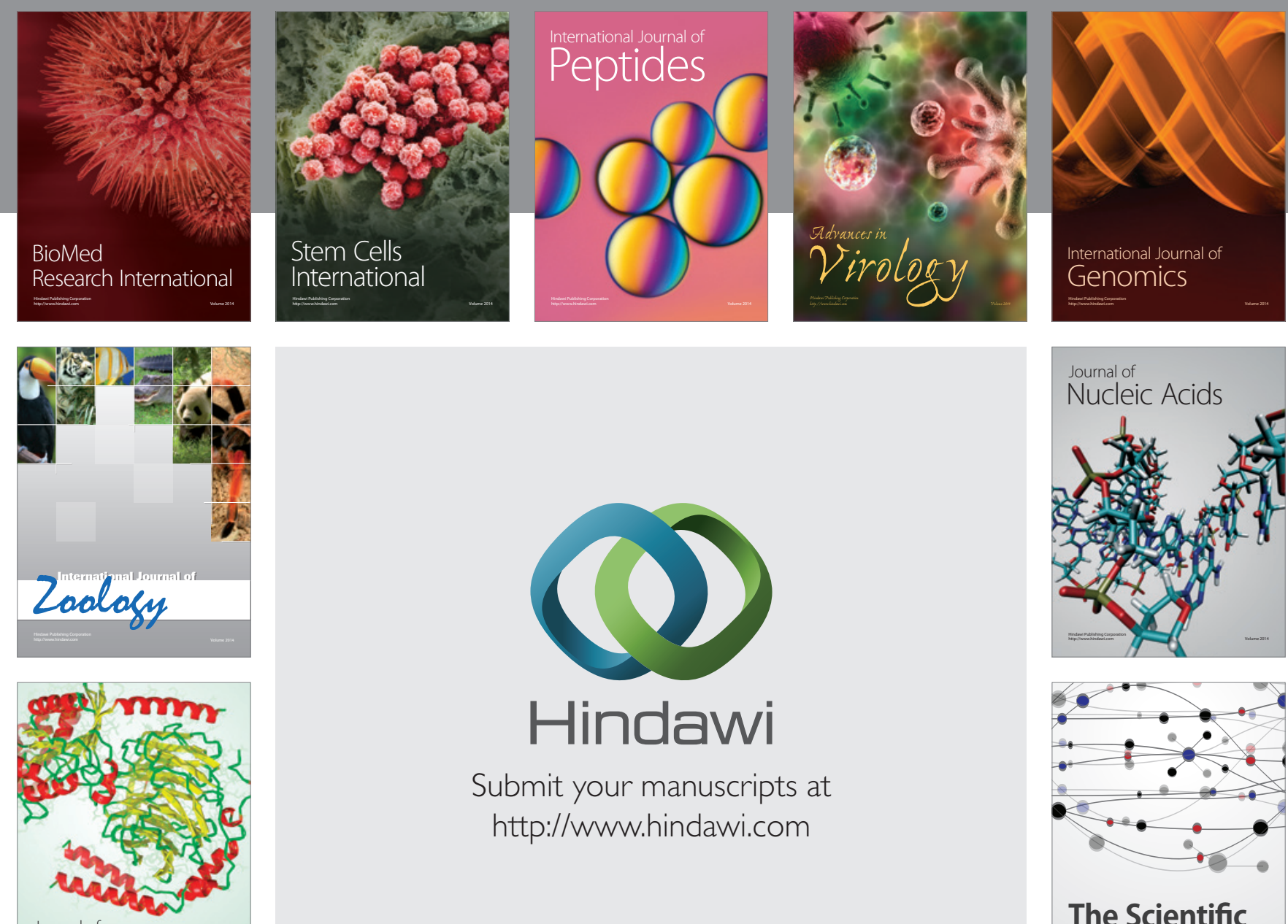

Submit your manuscripts at

http://www.hindawi.com

Journal of
Signal Transduction
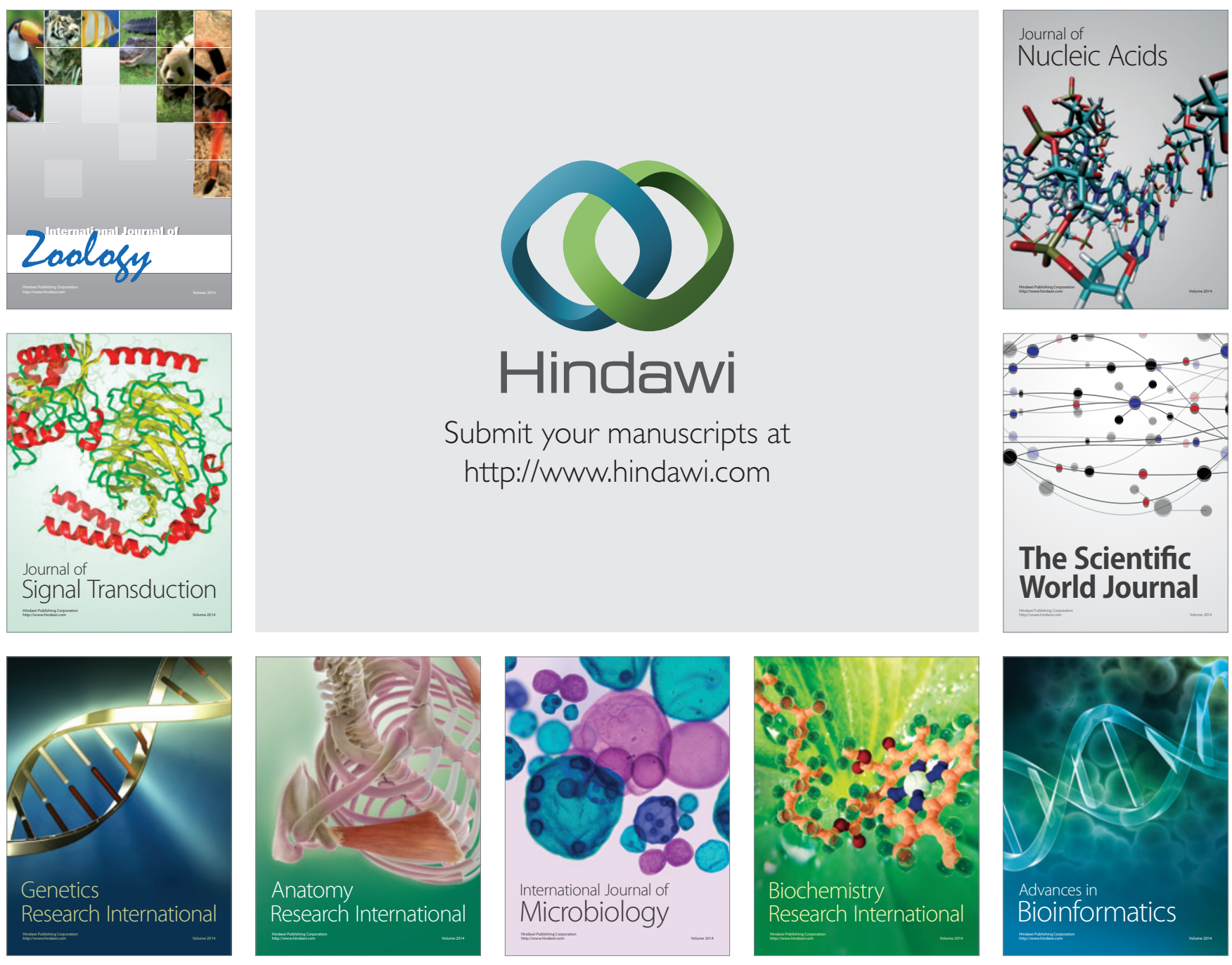

The Scientific World Journal
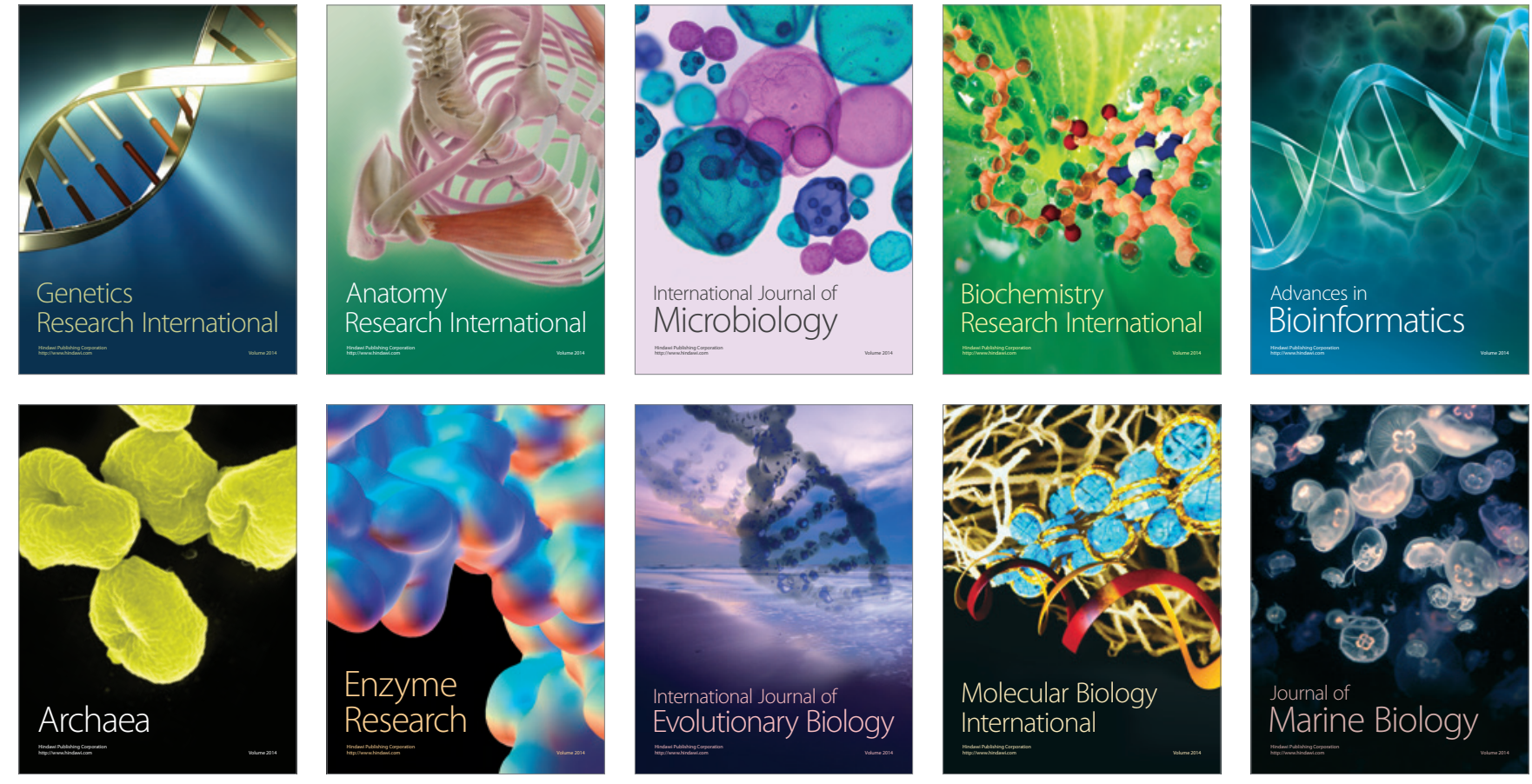